\title{
A LARGE ALGEBRAICALLY CLOSED FIELD
}

\author{
CLIFTON E. CORZATT ${ }^{1}$
}

ABSTRACT. A sequence is called an R.R.S. sequence if, roughly speaking, it is generated by some member of a set of recurrence formulas over the field $Q(i)$ which involves only rational operations. It is proved that the set of limits of all convergent R.R.S. sequences forms a countable algebraically closed field. Moreover, the field is shown to contain all numbers of the form $e^{a}$, where $a$ is an algebraic number.

Let $Q(i)$ denote the field of rationals with $i$ adjoined. We define an $m$ th order rational recursion scheme (R.R.S) to be one of the form $z_{1}(0), \cdots$, $z_{m}(0) \in Q(i)$ (initial conditions) and

$$
\begin{aligned}
z_{1}(n+1) & =R_{1}\left(z_{1}(n), \cdots, z_{m}(n)\right) \\
& : \\
z_{m}(n+1) & : R_{m}\left(z_{1}(n), \cdots, z_{m}(n)\right) \quad \text { (recursion formulae) }
\end{aligned}
$$

where $R_{1}, \cdots, R_{m}$ are rational functions of at most $m$ variables with coefficients from $Q(i)$. An R.R.S. sequence is one of the form $\left\{z_{j}(n)\right\}$ where $n=0,1,2, \cdots$ for some fixed $j$ with $1 \leq j \leq m$.

The set of all limits of convergent R.R.S. sequences forms a field which is denoted by $Q^{*}(i) . Q^{*}(i)$ is a countably infinite field, since the number of R.R.S. sequences is countable. We will prove that $Q^{*}(i)$ is algebraically closed, and that $Q^{*}(i)$ is large in that it contains all numbers of the form $e^{a}$ where $\alpha$ is an algebraic number. We note that the existence of fields like $Q^{*}(i)$ could be established by taking the algebraic closure of the rationals with all numbers of the form $e^{a}$ adjoined.

Proposition 1. $Q^{*}(i)$ is algebraically closed.

Proof. Let $P(z)=a_{r} z^{r}+a_{r-1} z^{r-1}+\cdots+a_{1} z+a_{0}$ be a polynomial

Received by the editors August 6, 1973.

AMS (MOS) subject classifications (1970). Primary 12F 20, $12 \mathrm{D} 99$.

Key words and phrases. Algebraic number, algebraically closed field.

${ }^{1}$ Research supported by NSF grant GP 28920. 
with coefficients in $Q^{*}(i)$ having $a$ as a root. Assume, without loss of generality, that $\alpha$ is a simple root. It suffices to show that $\alpha$ is in $Q^{*}(i)$.

Let $\left\{a_{j}(n)\right\}, n=0,1,2 \cdots$, be an R. R. S. sequence converging to $a_{j}$ where $0 \leq j \leq r$. Let $P_{n}(z)=a_{r}(n) z^{r}+a_{r-1}(n) z^{r-1}+\cdots+a_{0}(n)$. Note that $\left|a_{j}(0)-a_{j}\right|$ can be made arbitrarily small by adjusting the initial conditions of the R.R.S.

We will show that $z(n+1)=z(n)-\left(P_{n}(z(n)) / P_{n}^{\prime}(z(n))\right)$ is an R.R.S. sequence converging to $a$, subject to choosing $a_{j}(0)$ for $0 \leq j \leq r$ and $z(0)$ appropriately. (Here $P_{n}^{\prime}(z(n))$ is the derivative of $P_{n}(z(n))$ with respect to $z(n)$.)

Let $D$ be a disc with center $\alpha$ and radius less than or equal to $1 / 2^{k}$ with $k$ an integer greater than zero, so that $\left|P^{\prime}(z)\right|>2 m$ for $z \in D$ where $m$ is a positive real number.

Let

$$
\begin{aligned}
g_{n}(z)= & a_{r}(n)\left(z^{r-2}+z^{r-3}(z+\alpha)+\cdots+\left(z^{r-2}+z^{r-3} a+\cdots+a^{r-2}\right)\right) \\
& +a_{r-1}(n)\left(z^{r-3}+z^{r-4}(z+\alpha)+\cdots+\left(z^{r-3}+z^{r-4} a+\cdots+\alpha^{r-3}\right)\right) \\
& \cdot \\
& +a_{3}(n)(z+(z+\alpha))+a_{2}(n) .
\end{aligned}
$$

Now choose $a_{j}(0)$ for $0 \leq j \leq r$ so that $\left|P_{n}^{\prime}(z)\right| \geq m$ for $z \in D$ and $\left|P_{n}(\alpha)\right| /\left|P_{n}^{\prime}(z)\right| \leq 2^{-(k+s+4)}$ for $z \in D$, where $s$ is some positive integer so that $\left|g_{n}(z)\right| /\left|P_{n}^{\prime}(z)\right| \leq 2^{s}$ for $z \in D$, and choose $z(0)$ in $Q(i)$ so that $|z(0)-\alpha| \leq 2^{-(k+s+2)}$.

It is easy to show that

$$
z(n+1)-\alpha=(z(n)-\alpha)^{2} \cdot\left(g_{n}(z(n)) / P^{\prime}(z(n))\right)-P_{n}(\alpha) / P_{n}^{\prime}(z(n))
$$

for $n=0,1,2 \cdots$. So we have

$$
|z(n+1)-\alpha| \leq|z(n)-\alpha|^{2}\left|g_{n}(z(n)) / P_{n}^{\prime}(z(n))\right|+\left|P_{n}(\alpha) / P_{n}^{\prime}(z(n))\right|
$$

for $n=0,1,2 \cdots$. By induction, we have $|z(n)-\alpha| \leq 2^{-(k+s+3)}$ for $n \geq 1$. Since the sequence $\left\{P_{n}(\alpha)\right\}$ converges to zero, we can find an integer $N_{1}$ so that for $n \geq N_{1},\left|P_{n}^{n}(\alpha) / P_{n}^{\prime}(z(n))\right| \leq 2^{-(k+s+6)}$. It follows that $|z(n)-\alpha|$ $\leq 2^{-(k+s+5)}$ for $n \geq N_{1}$. This process can be repeated as often as one wants, so $\{z(n)\}$ converges to $a$. Thus $a$ is in $Q^{*}(i)$, and the proof of Proposition 1 is complete.

Now suppose $\alpha$ is an algebraic number of degree $n$. Since $Q^{*}(i)$ is 
algebraically closed we have that $\alpha$ is in $Q^{*}(i)$. We define rational numbers $b_{j}(k)$ for $0 \leq j \leq n-1$ to be the numbers which satisfy

$$
a^{k}=b_{0}(k)+b_{1}(k) \alpha_{+} \cdots+b_{n-1}(k) \alpha^{n-1} \quad \text { for } k=0,1,2, \cdots
$$

It is clear that $b_{0}(0)=1$ and that

$$
\alpha^{k+1}=b_{0}(k) \alpha+b_{1}(k) \alpha^{2}+\cdots+b_{n-2}(k) \alpha^{n-1}+b_{n-1}(k) \alpha^{n} .
$$

By substituting $\alpha^{n}=b_{0}(n)+b_{1}(n) a+\cdots+b_{n-1}(n) a^{n-1}$ in (1) we get

$$
\begin{aligned}
\alpha^{k+1}= & b_{n}(k) \cdot b_{0}(n)+\left(b_{0}(k)+b_{n-1}(k) b_{1}(n)\right) \alpha+\cdots \\
& +\left(b_{n-2}(k)+b_{n-1}(k) b_{n-1}(n)\right) \alpha^{n-1} .
\end{aligned}
$$

So if we assume that $b_{-1}(k)=0$ we get

$$
b_{j}(k+1)=b_{j-1}(k)+b_{n-1}(k) b_{j}(n)
$$

for $0 \leq j \leq n-1$ and $k=0,1,2, \cdots$.

From (2) we conclude that $b_{j}(1) \leq 2 M^{2}$ for $0 \leq j \leq n-1$ where $M=$ $\max \left\{1, b_{0}(n), \cdots, b_{n-1}(n)\right\}$ and by an induction

$$
b_{j}(k) \leq 2^{k} M^{k+1} \text { for } 0 \leq j \leq n-1 .
$$
$Q^{*}(i)$.

Proposition 2. If $a$ is an algebraic number of degree $n$ then $e^{a}$ is in

Proof. It is known that $e^{a}=\Sigma_{k=0}^{\infty} \alpha^{k} / k$ !. From (3) we get that $\Sigma_{k=0}^{\infty} b_{j}(k) / k$ ! is absolutely convergent so it is true that $e^{\dot{a}}=\sum_{j=0}^{n-1} a^{j} \sum_{k=0}^{\infty} b_{j}(k) / k$ !. Since $a$ is in $Q^{*}(i)$, if we can show that $\Sigma_{k=0}^{\infty} b_{j}(k) / k$ ! is in $Q^{*}(i)$ for $0 \leq j \leq n-1$ we will have $e^{a}$ in $Q^{*}(i)$. It suffices to show that there is an R.R.S. sequence converging to $\sum_{k=0}^{\infty} b_{j}(k) / k !$ for $0 \leq j \leq n-1$.

We exhibit an R.R.S. of order $3 n+4$ with initial conditions

$$
\begin{aligned}
z_{j}(0) & =b_{j}(0) \quad \text { for } 0 \leq j \leq n-1, \\
z_{j}(0) & =b_{j-n}(n) \quad \text { for } n \leq j \leq 2 n-1, \\
z_{2 n}(0) & =0, \\
z_{j}(0) & =b_{j-(2 n+1)}(1) \text { for } 2 n+1 \leq j \leq 3 n, \\
z_{3 n+1}(0) & =1, \quad z_{3 n+2}(0)=1, \quad z_{3 n+3}(0)=2,
\end{aligned}
$$

and recursion formulae 


$$
\begin{aligned}
z_{j}(k) & =z_{j}(k-1)+z_{j+2 n+1}(k-1) \cdot z_{3 n+2}(k-1) \quad \text { for } 0 \leq j \leq n-1, \\
z_{j}(k) & =z_{j}(k-1) \quad \text { for } n \leq j \leq 2 n-1, \\
z_{2 n}(k) & =z_{2 n}(k-1), \\
z_{k}(k) & =z_{j-1}(k-1)+z_{j-(n+1)}(k-1) \cdot z_{3 n}(k-1) \quad \text { for } 2 n+1 \leq j \leq 3 n, \\
z_{3 n+1}(k) & =z_{3 n+1}(k-1), \\
z_{3 n+2}(k) & =z_{3 n+2}(k-1) / z_{3 n+3}(k-1), \\
z_{3 n+3}(k) & =z_{3 n+3}(k-1)+z_{3 n+1}(k-1) .
\end{aligned}
$$

It can be shown by induction that

$$
\begin{aligned}
z_{j}(k) & =\sum_{m=0}^{k} \frac{b_{j}(m)}{m !} \text { for } 0 \leq j \leq n-1, \\
z_{j}(k) & =b_{j-n}(n) \text { for } n \leq j \leq 2 n-1, \\
z_{2 n}(k) & =b_{-1}(k+1)=0, \\
z_{j}(k) & =b_{j-(2 n+2)}(k)+b_{j-(2 n+1)}(n) \cdot b_{n-1}(k) \\
& =b_{j-(2 n+1)}(k+1) \text { for } 2 n+1 \leq j \leq 3 n, \\
z_{3 n+1}(k) & =1, \quad z_{3 n+2}(k)=\frac{1}{(k+1) !}, \quad z_{3 n+3}(k)=k+2 .
\end{aligned}
$$

Thus the sequence $\left\{z_{j}(k)\right\}$ converges to $\sum_{k=1}^{\infty} b_{j}(k) / k$ ! for $0 \leq j \leq$ $n-1$, and the sum is therefore in $Q^{*}(i)$. This completes the proof of Proposition 2.

The author wishes to thank Professor Kenneth.B. Stolarsky, his Ph.D. thesis advisor, for the suggestions which led to this research.

\section{REFERENCES}

1. C. Corzatt, A large algebraically closed field, Proc. 1972 Number Theory Conference, Boulder, Colorado, pp. 53-55.

2. C. Corzatt and K. B. Stolarsky, Sequences generated by rational operations, Proc. 1972 Number Theory Conference, Boulder, Colorado, pp. 228-232. 61801

DEPARTMENT OF MATHEMATICS, UNIVERSITY OF ILLINOIS, URBANA, ILLINOIS 